\title{
ARTICLE
}

Received 15 Dec 2014 | Accepted 19 Mar 2015 | Published 22 Apr 2015

\section{Highly compressible 3D periodic graphene aerogel microlattices}

Cheng Zhu' ${ }^{1}$, T. Yong-Jin Han ${ }^{1}$, Eric B. Duoss ${ }^{1}$, Alexandra M. Golobic ${ }^{1}$, Joshua D. Kuntz ${ }^{1}$, Christopher M. Spadaccini ${ }^{1} \&$ Marcus A. Worsley ${ }^{1}$

Graphene is a two-dimensional material that offers a unique combination of low density, exceptional mechanical properties, large surface area and excellent electrical conductivity. Recent progress has produced bulk 3D assemblies of graphene, such as graphene aerogels, but they possess purely stochastic porous networks, which limit their performance compared with the potential of an engineered architecture. Here we report the fabrication of periodic graphene aerogel microlattices, possessing an engineered architecture via a 3D printing technique known as direct ink writing. The 3D printed graphene aerogels are lightweight, highly conductive and exhibit supercompressibility (up to $90 \%$ compressive strain). Moreover, the Young's moduli of the 3D printed graphene aerogels show an order of magnitude improvement over bulk graphene materials with comparable geometric density and possess large surface areas. Adapting the 3D printing technique to graphene aerogels realizes the possibility of fabricating a myriad of complex aerogel architectures for a broad range of applications.

\footnotetext{
${ }^{1}$ Lawrence Livermore National Laboratory, 7000 East Avenue, Livermore, California 94550, USA. Correspondence and requests for materials should be addressed to M.A.W. (email: worsley1@llnl.gov).
} 
G raphene is an emerging class of ultrathin carbon membrane material ${ }^{1-3}$ with high specific surface area $^{4}$, superior elasticity ${ }^{5}$, chemical stability ${ }^{3}$ and high electrical and thermal conductivity ${ }^{6,7}$. These intrinsic physicochemical properties enable graphene to find widespread applications in nanoelectronics 8,9 , sensors ${ }^{10,11}$, catalysis ${ }^{12,13}$, composites $^{14,15}$, energy storage $\mathrm{e}^{6,17}$ and biomedical scaffolds ${ }^{18}$. To further explore various macroscopic applications of graphene materials, an essential prerequisite is controlled large-scale assembly of two-dimensional graphene building blocks and transfer of their inherent properties into three-dimensional (3D) structures. Template-guided methods, such as chemical vapour deposition coatings on metallic foams ${ }^{19}$ have been reported for the creation of $3 \mathrm{D}$ graphene monoliths, but the process is not scalable and the materials obtained from these methods are generally brittle under low compression ${ }^{20}$. Therefore, template-free approaches are still needed for scalable synthesis of 3D graphene macro-assemblies. Due to their simple and versatile synthesis scheme, and the ability to realize a wide range of pore morphologies, including ultrafine pore sizes $(<100 \mathrm{~nm})$, chemically derived graphene oxide (GO)-based aerogels are the most common $3 \mathrm{D}$ graphene found in the literature ${ }^{20-36}$. Starting from a widely available GO precursor, the main strategy to assemble porous $3 \mathrm{D}$ graphene networks is the self-assembly or gelation of the GO suspension via hydrothermal reduction ${ }^{20-22}$, chemical reduction ${ }^{27-31}$ or direct crosslinking ${ }^{33,34}$ of the GO sheets. Although some control over the pore morphology has been demonstrated with ice templating ${ }^{35,36}$, the architecture of these graphene networks remains largely random, precluding the ability to tailor transport and other mechanical properties of the material for specific applications (for example, separations, flow batteries, pressure sensors and so on) that might benefit from such engineering. Thus the fabrication of 3D graphene materials with tailored macro-architectures for specific applications via a controllable and scalable assembly method remains a significant challenge.

The functional characteristics of cellular materials are mostly determined by the intrinsic properties of their chemical composition, porosity and cell morphologies ${ }^{37}$. Several additive manufacturing techniques have been utilized to make highly ordered ultralight cellular materials possessing unique chemical, mechanical and structural properties by manipulation of their structures from the nanometre up to the centimetre scale ${ }^{38}$. For example, ultralight hollow metallic microlattices were produced using self-propagating photopolymer waveguide prototyping to form a template and subsequently coating the template with nickel-phosphorus via electroless plating ${ }^{39}$. Another example is the fabrication of ultralight, ultra-stiff octet truss metamaterials by the projection micro-stereolithography method ${ }^{40}$. However, these methods have limitations in their scaling and material diversity. Recently, an extrusion-based 3D printing technique, known as direct ink writing has also been applied to construct cellular elastomeric architectures ${ }^{41}$ and lightweight composites ${ }^{42}$. This technique employs a three-axis motion stage to assemble 3D structures by robotically extruding a continuous 'ink' filament through a micronozzle at room temperature in a layer-by-layer scheme $e^{43}$. The primary challenge for this method is to design gel-based viscoelastic ink materials possessing shear thinning behaviour to facilitate flow under pressure and a rapid pseudoplastic to dilatant recovery after deposition resulting in shape retention ${ }^{44,45}$. Although a number of ceramic, metallic, polymeric and even graphene-polymer composite ink materials ${ }^{43,46,47}$ have been developed to fabricate various complex 3D structures, there is no example using this technique to create $3 \mathrm{D}$ periodic graphene aerogel macroarchitectures.
In this work, we demonstrate a $3 \mathrm{D}$ printing strategy for the fabrication of 3D graphene aerogels with designed macroscopic architectures. Our approach is based on the precise deposition of GO ink filaments on a pre-defined tool path to create architected $3 \mathrm{D}$ structures. Two key challenges in this process are developing a printable graphene-based ink and maintaining the intrinsic properties of single graphene sheets (for example, large surface area, mechanical and electrical properties) in the $3 \mathrm{D}$ printed structures. To this end, we have developed a new GO-based ink and printing scheme that allows the manufacture of porositytunable hierarchical graphene aerogels with high surface area, excellent electrical conductivity, mechanical stiffness and supercompressibility.

\section{Results}

Three-dimensional printing of graphene aerogels. The first challenge for this fabrication strategy is to develop printable GO inks, by tailoring the composition and rheology required for reliable flow through fine nozzles, and self-supporting shape integrity after deposition (for example, highly viscous, nonNewtonian fluids). Printable GO ink development is challenging because most GO-based graphene aerogels begin with fairly dilute precursor GO suspensions $\left(<5 \mathrm{mg} \mathrm{ml}^{-1} \mathrm{GO}\right)$ that do not possess the required rheological behaviour for a $3 \mathrm{D}$ printable ink as they are low-viscosity $(\eta)$ Newtonian fluids ${ }^{20,48}$. Recently, the rheological behaviour of GO dispersions has been investigated to enable further fabrication of $\mathrm{GO}$ into complex architectures ${ }^{49}$. There are reports of higher concentration GO suspensions (for example, $10-20 \mathrm{mg} \mathrm{ml}^{-1} \mathrm{GO}$ ) that can also make high-quality graphene aerogels ${ }^{30,33}$. These reports demonstrate gelation of concentrated GO suspensions under basic conditions (for example, addition of ammonium hydroxide) or direct crosslinking using organic sol-gel chemistry (for example, resorcinolformaldehyde (R-F) solution). As the gelation method can influence the aerogel microstructure ${ }^{34}$, both methods were applied to the high-concentration GO suspensions we investigated for the GO inks. Figure 1a shows the apparent viscosity of high-concentration GO suspensions as a function of shear rate, revealing that at $20 \mathrm{mg} \mathrm{ml}^{-1}$, the GO suspension shows orders of magnitude higher apparent viscosity than reported at lower concentrations ${ }^{48}$, and that the GO suspension at $20 \mathrm{mg} \mathrm{ml}^{-1}$ is a shear-thinning non-Newtonian fluid, which is necessary for a printable ink. Further increasing the GO concentration to $40 \mathrm{mg} \mathrm{ml}^{-1}$ results in another order-ofmagnitude increase in apparent viscosity, which further improves printability. Finally, addition of hydrophilic fumed silica powders to the GO suspensions imparts additional increases in viscosity. Silica filler serves as a removable viscosifier by imparting both shear thinning behaviour and a shear yield stress to the GO suspension to further enhance the printability of the GO inks. Figure $1 \mathrm{~b}$ compares the pure GO suspensions and representative GO inks storage and loss moduli with varying compositions. Specifically, the pure $20-\mathrm{mg} \mathrm{ml}^{-1} \mathrm{GO}$ suspensions without fillers exhibit a plateau value of its elastic modulus $\left(G^{\prime}\right)$ $\sim 1,000 \mathrm{~Pa}$ and a yield stress $\left(\tau_{y}\right) \sim 40 \mathrm{~Pa}$, respectively. By adding $20 \mathrm{wt} \%$ silica powders into pure $20 \mathrm{mg} \mathrm{ml}^{-1} \mathrm{GO}$ suspensions, both elastic modulus and yield stress increase by approximately an order of magnitude. Meanwhile, the addition of $10 \mathrm{wt} \%$ silica filler increases the elastic modulus and yield stress of $40 \mathrm{mg} \mathrm{ml}^{-1}$ GO suspensions by over an order magnitude. The magnitudes of these key rheological parameters are in good agreement with those reported for other colloidal inks designed for this 3D filamentary printing technique ${ }^{45}$. Although the pure $40-\mathrm{mg} \mathrm{ml}^{-1}$ GO suspension ink is printable, the silica-filled GO inks were preferred due to their superior rheological properties and facile 


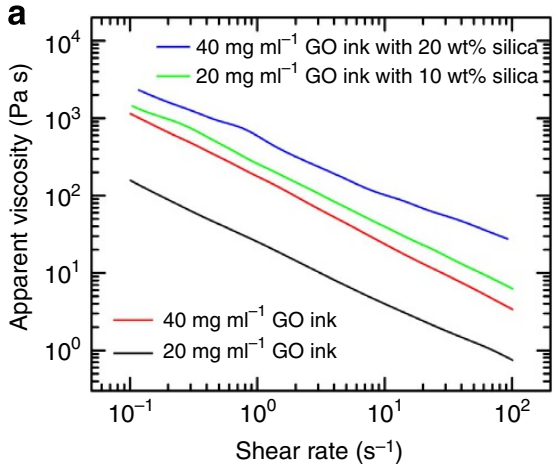

C
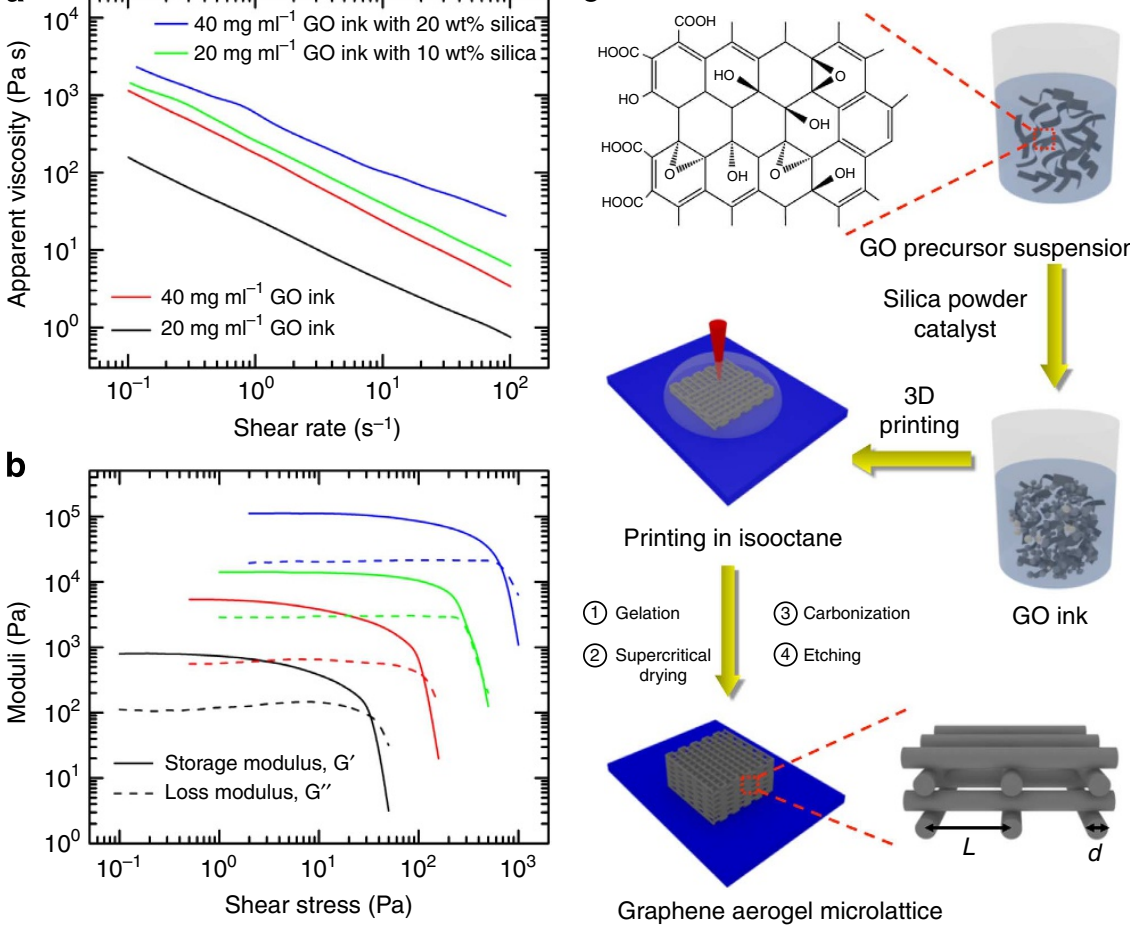

Figure 1 | Fabrication strategy and GO ink's rheological properties. Log-log plots of (a) apparent viscosity as a function of shear rate and (b) storage and loss modulus as a function of shear stress of $\mathrm{GO}$ inks with and without silica fillers. (c) Schematic of the fabrication process. Following the arrows: fumed silica powders and catalyst (that is, $\left(\mathrm{NH}_{4}\right)_{2} \mathrm{CO}_{3}$ or $\mathrm{R}-\mathrm{F}$ solution) were added into as-prepared aqueous $\mathrm{GO}$ suspensions. After mixing, a homogeneous $\mathrm{GO}$ ink with designed rheological properties was obtained. The $\mathrm{GO}$ ink was extruded through a micronozzle immersed in isooctane to prevent drying during printing. The printed microlattice structure was supercritically dried to remove the liquid. Then, the structure was heated to $1,050^{\circ} \mathrm{C}$ under $\mathrm{N}_{2}$ for carbonization. Finally, the silica filler was etched using HF acid. The in-plane centre-to-centre rod spacing is defined as $L$, and the filament diameter is defined as $d$.

removal of the silica during post-processing. In addition to these, GO inks exhibit the desired viscoelasticity and they have a long pot life.

The process of $3 \mathrm{D}$ printing the GO inks such that a $3 \mathrm{D}$ graphene aerogel structure is produced also presents several obstacles. Aerogels are ultralow-density porous solids created by carefully replacing the liquid in the pores of the wet gel with air. To convert the $3 \mathrm{D}$ printed GO structure to an aerogel, the GO ink must remain wet through printing and gelation such that the liquid in the GO gel can be removed via supercritical- or freezedrying to avoid gel collapse due to capillary forces. This necessitates printing the GO ink into a bath of liquid that is not only less dense than water but immiscible with our aqueous GO inks. The fabrication scheme for accomplishing this is illustrated in Fig. 1c. An animation of the fabrication scheme used to print the graphene aerogel microlattices can also be seen in Supplementary Movie 1 . The GO inks are prepared by combining a GO suspension and silica filler to form a homogenous, highly viscous and thixotropic ink. These GO inks are then loaded into a syringe barrel and extruded through a micronozzle to pattern $3 \mathrm{D}$ structures. To prevent the ink from drying in the air, which can clog the tip of the printing apparatus or cause pore collapse in the printed structure, the printing is carried out in an organic solvent bath (isooctane) that is not miscible with the aqueous ink. Finally, the printed structures can be processed according to standard literature methods ${ }^{29,30}$, followed by etching of the silica filler to obtain the ultimate periodic 3D graphene aerogel microlattices.

To demonstrate $3 \mathrm{D}$ printing of graphene aerogels, we first printed woodpile, 'simple cubic'-like lattices consisting of multiple orthogonal layers of parallel cylindrical filaments successively printed in a layer-by-layer fashion. These 3D simple cubic lattices are designed with an in-plane centre-to-centre filament spacing $(L)$ of $1 \mathrm{~mm}$ and a filament diameter $(d)$ of $0.25 \mathrm{~mm}$, resulting in a spacing-to-diameter ratio $(L / d)$ of 4 (Fig. $1 c$ ). By simply changing the filament spacing and diameter, we have the ability to $3 \mathrm{D}$ print graphene structures over a wide range of geometric densities. The printed 3D graphene aerogel microlattice shows excellent structural integrity and micro-architecture accuracy (Fig. 2a,b), which is indicative of the high quality of the ink material for this printing process (see Supplementary Movie 2). After the removal of silica fillers (Supplementary Fig. 1a), there are random large pores distributed in graphene aerogels (Fig. 2c,d; Supplementary Fig. 1b). Figure $2 \mathrm{c}, \mathrm{d}$ also shows how the microstructure of the $3 \mathrm{D}$ printed graphene aerogels can be tuned by simply modifying the GO ink formulations. Similar to results observed in bulk monolithic graphene aerogels ${ }^{34}$, changes in the gelation chemistry can lead to significant microstructural changes. In this case, we use either basic solution (for example, $\left(\mathrm{NH}_{4}\right)_{2} \mathrm{CO}_{3}$ ) to directly crosslink graphene sheets via the functional groups (for example, epoxide and hydroxide) or resorcinol (R) and formaldehyde (F) with sodium carbonate as a catalyst to 'glue' the sheets together. The use of organic sol-gel chemistry ( $\mathrm{R}-\mathrm{F}$ solution) to build the GO network led to a more open, less crosslinked network (Fig. 2d) compared with gelation methods based on GO's native functionality (that is, no R-F) (Fig. 2c). The ability to tune the microstructure, in addition to the macrostructure, is important because it can affect a wide range of properties such as density, conductivity, surface area and, as noted below, mechanical properties. This approach opens new opportunities for the fabrication of graphene-based structures at the macroscale. To further demonstrate the flexibility of this $3 \mathrm{D}$ printing technique, we fabricated a series of graphene aerogel 
microlattices with varying thicknesses and a large area graphene aerogel honeycomb (Fig. 2e,f).

Physical properties of 3D printed graphene aerogels. Modifying the GO suspensions to make suitable inks has the potential to alter the properties of the final aerogel; however, most properties of the 3D printed graphene aerogels were found to meet or exceed those of the bulk material. For example, techniques such as Raman spectroscopy, X-ray diffraction (XRD) and energy-dispersive X-ray spectroscopy (EDS) were applied to see how microstructure, graphene layering and degree of GO reduction compare with bulk graphene aerogels. Raman spectra of the 3D
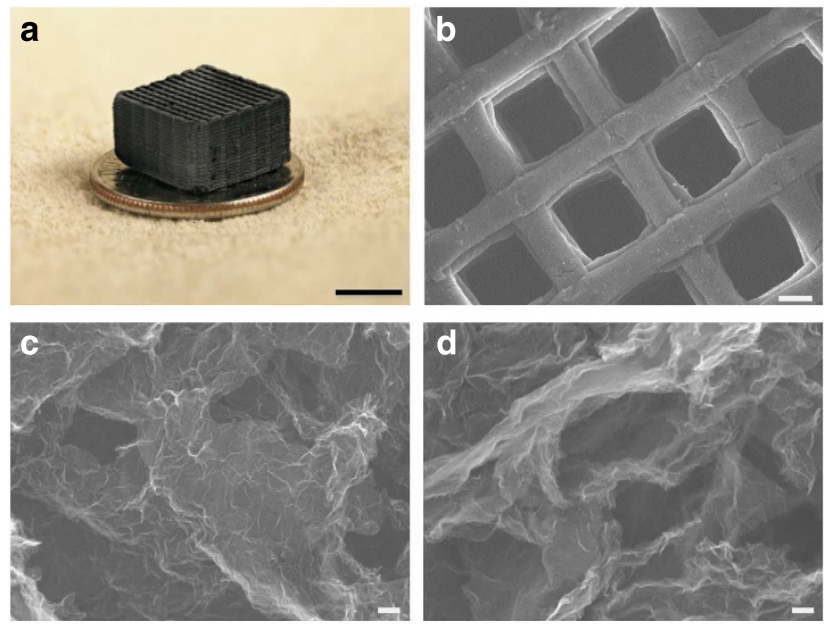

e

f
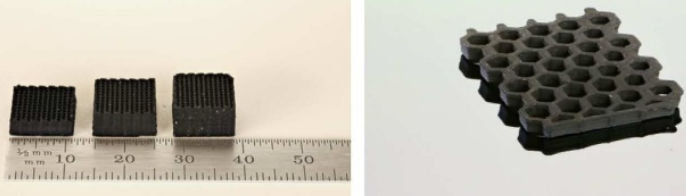

Figure 2 | Morphology and structure of graphene aerogels. (a) Optical image of a 3D printed graphene aerogel microlattice. SEM images of (b) a 3D printed graphene aerogel microlattice, (c) graphene aerogel without $\mathrm{R}-\mathrm{F}$ after etching and (d) graphene aerogel with $4 \mathrm{wt} \% \mathrm{R}-\mathrm{F}$ after etching. Optical image of (e) 3D printed graphene aerogel microlattices with varying thickness and (f) a 3D printed graphene aerogel honeycomb. Scale bars, $5 \mathrm{~mm}(\mathbf{a}), 200 \mu \mathrm{m}(\mathbf{b}), 100 \mathrm{~nm}(\mathbf{c}, \mathbf{d}), 1 \mathrm{~cm}(\mathbf{f})$. printed graphene aerogels (Fig. 3a) all show strong D $\left(1,350 \mathrm{~cm}^{-1}\right)$ and $\mathrm{G}\left(1,582 \mathrm{~cm}^{-1}\right)$ bands with weak, broad $\mathrm{D}^{\prime}$ and $\mathrm{G}^{\prime}$ features identical to those previously reported for bulk aerogels ${ }^{29,30}$, suggesting a similar microstructure and defect density. XRD spectra of 3D printed graphene aerogels (Fig. 3b) are also similar to those of bulk graphene aerogels ${ }^{29,30}$, showing weak, broad features indicative of single- and few-layer graphene. EDS (Supplementary Fig. 2) also shows that, like the bulk graphene aerogel, the $\mathrm{C}: \mathrm{O}$ ratio of $3 \mathrm{D}$ printed graphene aerogel rises to $>20$ compared with 5 for the native GO, confirming a high level of GO reduction. EDS also confirms that the silica filler has been completely removed from the graphene microlattice. Together, the scanning electron microscopy (SEM), Raman, XRD and EDS show that the $3 \mathrm{D}$ printed graphene aerogel is quite similar to the bulk graphene aerogel and is not significantly degraded by the etching or printing process.

Standard graphene aerogels are also notable for their large surface areas, low densities and high electrical conductivities. These characteristics are also evaluated for the modified formulations that we used to create the inks and are presented in Table 1. Nitrogen porosimetry (Table 1; Supplementary Fig. 3) show that the modified formulations maintain a high surface area (700-1,100 $\left.\mathrm{m}^{2} \mathrm{~g}^{-1}\right)$ and large mesopore volumes $\left(2-4 \mathrm{~cm}^{3} \mathrm{~g}^{-1}\right)$, consistent with the SEM images and comparable to bulk graphene aerogels in the literature ${ }^{29,30}$. Four-probe and density measurements also show that the modified formulations retain a low density and high conductivity characteristic of standard graphene aerogels. As seen in previous reports ${ }^{29,30}$, all these properties (surface area, conductivity and density) can be tuned by changing the $\mathrm{R}-\mathrm{F}$ concentration in the initial suspension. The GO concentration also appears to impact the surface area of the aerogel. The slightly lower surface areas at higher GO concentrations likely stem from larger fractions of few-layer graphene due to less efficient exfoliation.

Graphene aerogels are also known to be remarkably stiff and flexible. To quantify the mechanical properties of these aerogels, we conducted in-plane compression tests to measure the compressive stress $(\sigma)$ as a function of strain $(\varepsilon)$ for all bulk and printed structures. The compressibility of these graphene aerogels is displayed in Fig. 4. It presents the stress-strain curves of five stepped compression cycles with strain amplitude of 10,20, 30,40 and $50 \%$ in sequence. The starting point for each cycle is the same and equal to the initial thickness of the sample, no matter how much unrecoverable compression is in the previous cycle. It is interesting that each succeeding loading curve exactly rises back to the maximum stress-strain point of the preceding cycle and continues the trend of the preceding loading curve in the full range of our measurements, showing a perfect strain
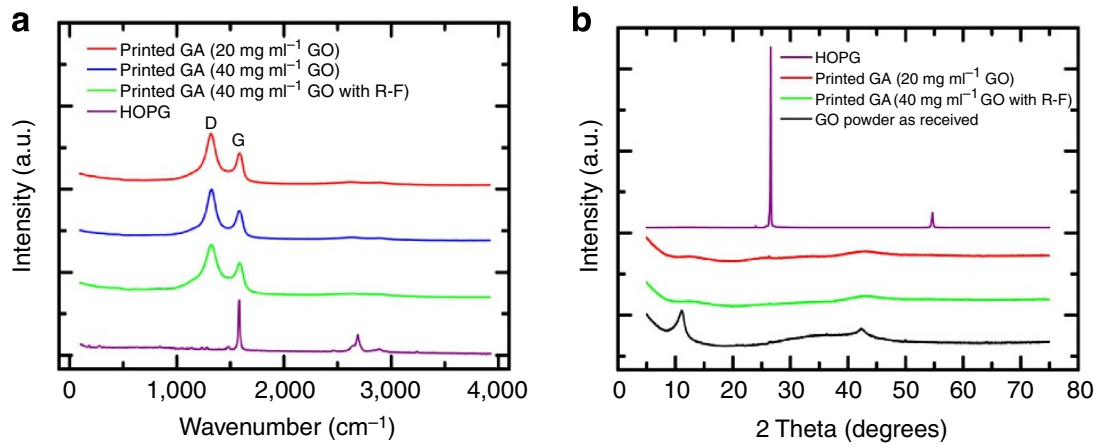

Figure 3 Raman and XRD spectra of graphene aerogels. (a) Raman and (b) XRD spectra of 3D printed graphene aerogel microlattices made with various ink formulations. Spectra of highly oriented pyrolytic graphite (HOPG) and graphene oxide (GO) powder are included for reference. 
memory effect. Figure 4a,b shows the stress-strain curves of bulk and printed graphene aerogels using the native functionality of the GO sheets with loading curves that display linear elastic properties from 10 to 50\% strain. From the unloading curves, we can find each compression leads to a degree of permanent residual deformation, and the recoverability of the printed aerogels is slightly higher than that of bulk aerogels.

In contrast, bulk and printed graphene aerogels using GO inks with organic sol-gel chemistry to crosslink GO sheets exhibit extraordinary supercompressibility, with full recovery after large strains (Fig. 4c,d). As the main difference between the aerogels lies in their microstructure (Fig. $2 c, d)^{34}$, we propose that the difference in compressive behaviour is linked to their microstructural differences. The loading curves of both bulk and printed aerogels show three distinct regions typically observed in other cellular materials, namely an initial Hookean region at $5 \%<\varepsilon<10 \%$, a plateau at $10 \%<\varepsilon<40 \%$ and a densification regime for $\varepsilon>40 \%$ with a steep increase in stress. Thus, similar to other resilient cellular materials ${ }^{36,37}$, hysteresis loops are found in the loading-unloading cycles, which indicate

\section{Table 1 | Physical properties of different 3D printed} graphene aerogel formulations.

\begin{tabular}{|lcccc} 
Ink formulation & $\begin{array}{c}\text { Aerogel } \\
\text { density } \\
\left(\mathbf{m g ~ c m}^{-3} \mathbf{)}\right)\end{array}$ & $\begin{array}{c}\text { BET } \\
\text { surface } \\
\text { area } \\
\left(\mathbf{m}^{\mathbf{2}} \mathbf{g}^{-\mathbf{1}} \mathbf{)}\right.\end{array}$ & $\begin{array}{c}\text { Pore } \\
\text { volume } \\
\left(\mathbf{c m}^{\mathbf{3}} \mathbf{g}^{-\mathbf{1}}\right)\end{array}$ & $\begin{array}{c}\text { Conductivity } \\
\left(\mathbf{S ~ m}^{-\mathbf{1}}\right)\end{array}$ \\
\hline $20 \mathrm{mg} \mathrm{ml}^{-1} \mathrm{GO}$ & 31 & 1,066 & 4.1 & 87 \\
$40 \mathrm{mg} \mathrm{ml}^{-1} \mathrm{GO}$ & 60 & 955 & 3.8 & 198 \\
$40 \mathrm{mg} \mathrm{ml}^{-1} \mathrm{GO}$ & 123 & 704 & 2.5 & 278 \\
with R-F & & & & \\
\hline
\end{tabular}

BET, Brunauer-Emmett-Teller; 3D, three-dimensional; GO, graphene oxide; R-F, resorcinolformaldehyde. energy dissipation that can be attributed to the buckling of microstructures, the friction and adhesion between branches and the cracks that occur primarily in the first compression for the large dissipation. The initial increase of stress in the range of $\varepsilon<5 \%$ is attributed to the increase of contact area between the sample and the platen for our compression fixture. The primary deformation in the Hookean region is linear elastic dominated by bending mode deformation. The plateau is mainly attributed to the buckling deformation of the graphene sheets. As the graphene aerogel crosslinked via organic sol-gel chemistry has a more open, less crosslinked microstructure and the graphene sheets are free to bend and buckle under compression, there is substantial recovery when the load is removed. Even after compression to 90\% strain, less than 5\% residual deformation was observed (Supplementary Fig. 4).

To further assess and characterize the stability of the cyclic resilient property of printed graphene aerogels, compression cycling of the graphene aerogel at $50 \%$ strain was conducted (Fig. 5). Energy dissipation is one of the key functions of cellular materials, and our printed graphene elastomers exhibit excellent energy absorption capability. In Fig. 5a,b, the energy loss coefficient of printed aerogels decreases from 60 to $30 \%$ in the first three cycles and then remains fairly constant. The maximum stress for each cycle in Fig. 5a also shows a similar trend (Fig. 5b). The electrical resistance of the printed graphene aerogels was also determined as a function of cyclic compression (Fig. 5c). The electrical resistance of the printed aerogels shows only a slight decrease after multiple compression events, confirming the remarkable structural resilience of the graphene aerogel microlattices.

Finally, the effect of macroscopic architectural design on the mechanical properties is also reflected in superior rigidity of the graphene aerogel microlattices compared with bulk aerogels at the same overall geometric density. It has been shown that the stiffness of many conventional cellular solids is significantly diminished as their densities decrease due to quadratic or higher
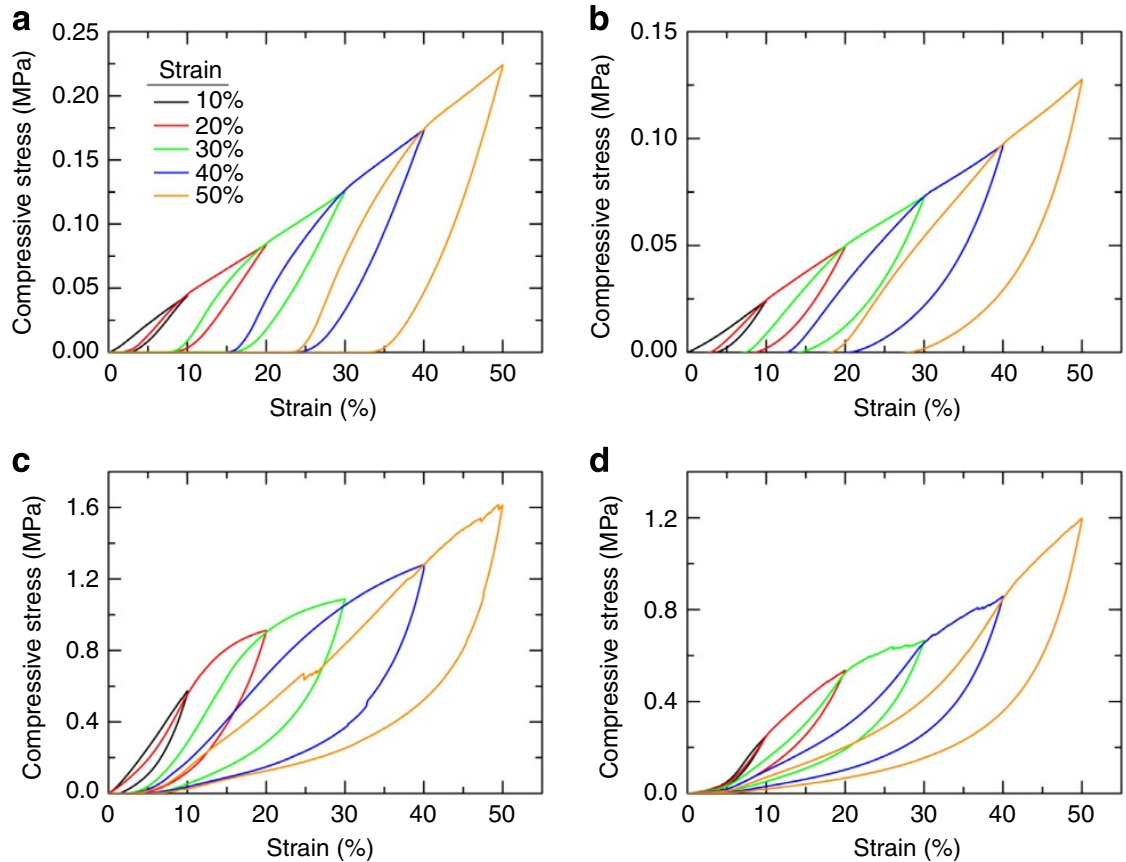

Figure 4 | Compressive properties of graphene aerogels. Stress-strain curves during loading-unloading cycles in sequence of increasing strain amplitude for (a) bulk graphene aerogel $\left(31 \mathrm{mg} \mathrm{cm}^{-3}\right)$ and (b) 3D printed graphene aerogel microlattice $\left(16 \mathrm{mg} \mathrm{cm}^{-3}\right)$ using the GO ink without R-F, (c) bulk graphene aerogel $\left(123 \mathrm{mg} \mathrm{cm}^{-3}\right)$ and (d) $3 \mathrm{D}$ printed graphene aerogel microlattice $\left(53 \mathrm{mg} \mathrm{cm}^{-3}\right)$ using the GO ink with R-F. 
a

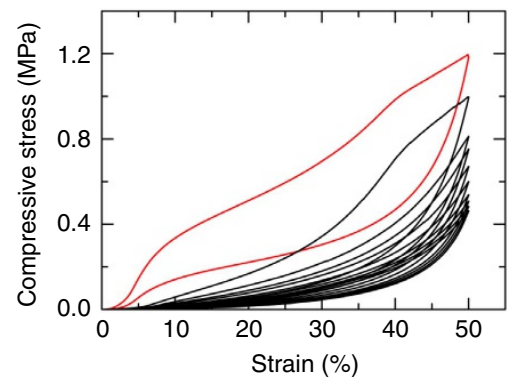

C

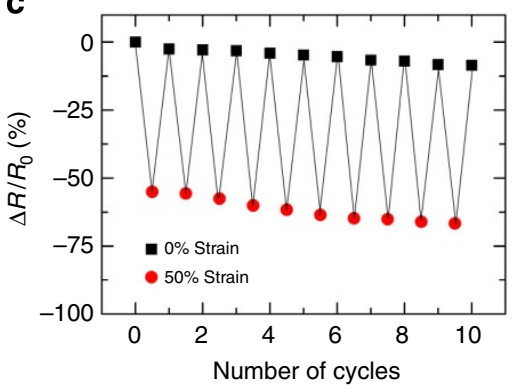

b
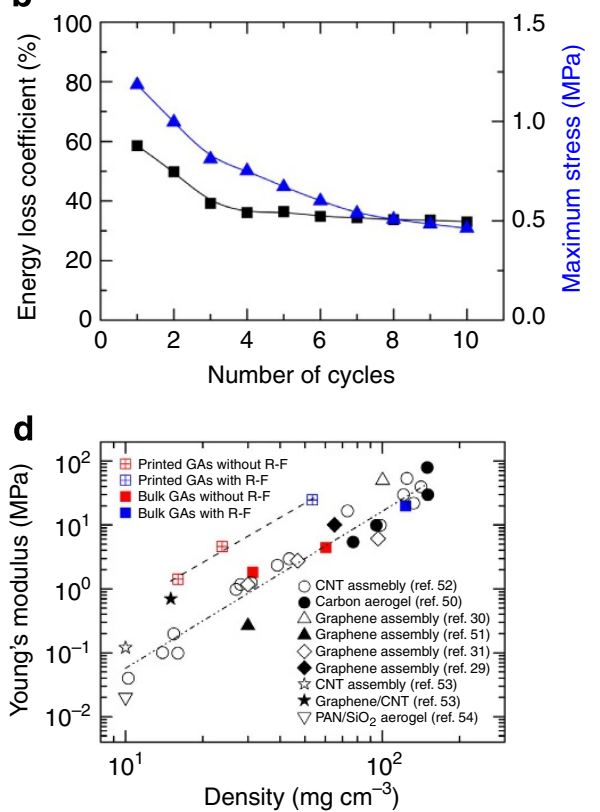

Figure 5 | Physical properties of graphene aerogels. (a) Compressive stress-strain curves of 10 cycles of loading-unloading. (b) Maximum stress and energy loss coefficient during 10 cycles. (c) Electrical resistance change when repeatedly compressed up to $50 \%$ of strain for 10 cycles. The graphene aerogel microlattice used for cyclic compression and conductivity measurements (a-c) has a geometric density of $53 \mathrm{mg} \mathrm{cm}^{-3}$. (d) The relationships between Young's modulus and density of bulk and printed graphene aerogels.

power scaling relationships between Young's modulus $(E)$ and density $(\rho)^{39}$. Figure $5 \mathrm{~d}$ shows the Young's modulus as a function of density for our graphene microlattices (printed) and standard graphene aerogels (bulk) compared against other carbon, carbon nanotube and graphene assemblies found in the literature $29-31,50-54$ as a function of density. The bulk aerogel data are consistent with literature data, while the printed aerogel data are substantially offset from the known curve. The log-log plot in each case demonstrates the expected power-law density dependence of Young's modulus. In both cases, Young's modulus was found to scale with density as $E \propto \rho^{2.5}$, consistent with other studies ${ }^{29}$. The similar values of the exponent $(\sim 2.5)$ indicate both printed and bulk aerogels show the same bendingdominated behaviour under compression. However, the magnitude of $E$ for the graphene microlattices was about one order of magnitude larger than that of most bulk graphene aerogels with same densities. In other words, the printed graphene aerogels can maintain the stiffness values of higherdensity bulk aerogels to much lower densities. This phenomenon is also commonly observed in traditional cellular materials, such as honeycombs, which exhibit stiffness values in certain loading directions that rival higher-density bulk solids ${ }^{37}$. In the case of the printed aerogels, the printed structures exhibit Young's moduli that rival bulk aerogel values with nearly twice the density of their printed counterparts. Upon closer inspection, it is revealed that the Young's modulus of the printed structure is approximately equal to that of the bulk aerogel with the same density as that of the printed aerogel filaments within the lattice. Thus, the improved performance is primarily attributed to the local density in the printed aerogels rather than the overall density, which accounts for the macroscale pores. In other words, the stiffness is controlled by the density of each filament, which is much higher than the geometric density of total microlattice. In fact, the actual geometric density of the printed aerogel is quite consistent with the theoretically expected value for each lattice (Supplementary Fig. 5). These observations show that the introduction of periodic macroscale pores in the $3 \mathrm{D}$ printed microlattices can provide additional functionality to the aerogel (for example, lower density, faster mass transport and so on) with negligible impact on the mechanical integrity of structure. Thus, the 3D printed graphene aerogels would benefit technologies such as catalysis, desalination and other filtration/ separation applications that require large surface areas, low density, superior mechanical rigidity and engineered porosity for predictable fluid flow characteristics.

\section{Discussion}

We present a general strategy for fabrication of periodic graphene aerogel microlattices via 3D printing. Key factors for successful $3 \mathrm{D}$ printing of aerogels included modifying GO precursor suspension such that it serves as printable ink, and adapting the $3 \mathrm{D}$ printing process to prevent premature drying of the printed structure. By addressing these issues, 3D printed aerogel microlattices were produced with properties that met or exceeded those of bulk aerogel materials. The graphene microlattices possess large surface areas, good electrical conductivity, low relative densities and supercompressibility, and are much stiffer than bulk graphene of comparable geometric density. By modifying the microstructure and density of the graphene aerogel through changing the ink formulation, we also showed how mechanical properties of the microlattices can be tuned. As graphene aerogels are currently being explored for a broad range of applications, having a manufacturing method for creating periodic or engineered structures using this novel material will further expand the range of applications where graphene can be utilized. In particular, our strategy makes it possible to explore the properties and applications of graphene in a self-supporting, structurally tunable and 3D macroscopic form. This work presents a versatile method for fabricating a broad class of $3 \mathrm{D}$ macroscopic graphene aerogel structures of determined geometries, and could lead to new types of graphene-based electronics, 
energy storage devices, catalytic scaffolds and separation devices. Furthermore, other functional materials can be readily incorporated into the open void space, offering opportunity to create new graphene-based nanocomposites. Finally, this fabrication scheme could be broadly applied to other aerogel systems enabling 3D printed aerogel structures for the myriad of technologies that require high surface area, low-density materials.

\section{Methods}

GO ink preparation. Raw single-layer GO powder (Cheaptubes) was produced by the Hummer method ${ }^{55}$ and had lateral dimensions of $300-700 \mathrm{~nm}$. GO suspensions were prepared via ultrasonication at concentrations of 20 and $40 \mathrm{mg} \mathrm{ml}^{-1}$ in water for $24 \mathrm{~h}$ in a VWR Scientific Model 57T Aquasonic (sonic power $\sim 90 \mathrm{~W}$, frequency $\sim 40 \mathrm{kHz}$ ). After sonication, the lateral dimensions were in the range of $150-400 \mathrm{~nm}$. For GO inks gelled using the native functionality on the $\mathrm{GO}$ sheets, ammonium carbonate $\left(\left(\mathrm{NH}_{4}\right)_{2} \mathrm{CO}_{3}\right)$ solution $(0.3 \mathrm{wt} \%)$ was used. In a typical synthesis, GO inks are prepared by mixing $6 \mathrm{~g}$ of $40 \mathrm{mg} \mathrm{ml}^{-1} \mathrm{GO}$ suspension, $0.343 \mathrm{~g}\left(\mathrm{NH}_{4}\right)_{2} \mathrm{CO}_{3}$ solution and $0.7 \mathrm{~g}$ fumed silica (EH-5, Cabot), respectively. For GO inks gelled using organic sol-gel chemistry, the sol-gel mixture consisted of an aqueous solution of resorcinol (R), formaldehyde (F) and sodium carbonate catalyst (C). The R:F mole ratio was $1: 2$, the $R: C$ mole ratio was 200:1 and the resultant R-F concentration was $11 \mathrm{wt} \% \mathrm{R}-\mathrm{F}$ solids. In a typical synthesis, $3.6 \mathrm{~g}$ of $40 \mathrm{mg} \mathrm{ml}^{-1} \mathrm{GO}$ suspensions, $2 \mathrm{~g}$ of R-F solution and $0.7 \mathrm{~g}$ of fumed silica are mixed. A planetary centrifugal mixer (Thinky) was used for mixing these samples for $2 \mathrm{~min}$ in a $35-\mathrm{ml}$ container using a custom adaptor.

Ink rheology. Rheological properties of the inks were characterized using a stresscontrolled Rheometer (AR 2000ex, TA) with a 40-mm-flat plate geometry and a gap of $500 \mu \mathrm{m}$. All measurements were preceded by a 1-min conditioning step at a constant shear rate of $1 \mathrm{~s}^{-1}$, followed by a 10-min rest period to allow the ink structure to reform. A stress sweep from $10^{-2}$ to $10^{3} \mathrm{~Pa}$ at a constant frequency of $1 \mathrm{~Hz}$ was conducted to record the storage modulus and loss modulus variations as a function of sweep stress. The yield stress $\left(\tau_{y}\right)$ was defined as the stress where storage modulus falls to $90 \%$ of the plateau value. A strain sweep from $10^{-1}$ to $10^{2} \mathrm{~s}^{-1}$ was also performed to record the apparent viscosity at varying shear rates.

3D printing. The GO ink was housed in a $3 \mathrm{ml}$ syringe barrel (EFD) attached by a luer-lok to a smooth-flow tapered nozzle $(250 \mu \mathrm{m}$ inner diameter, $d)$. An airpowered fluid dispenser (Ultimus V, EFD) provided the appropriate pressure to extrude the ink through the nozzle. The target patterns were printed using a threeaxis positioning stage (ABL 9000, Aerotech The 3D GO structures were printed onto silicon wafers in an isooctane (2,2,4-trimethylpentane) bath, with an initial nozzle height of $0.7 d$ to ensure adhesion to the substrate. Three-dimensional periodic microlattices were assembled by patterning an array of parallel (rod-like) filaments in a meanderline-like pattern in the horizontal plane such that the orientation of each successive layer was orthogonal to the previous layer. Threedimensional honeycomb structures were fabricated by stacking hexagonal unit-cell arrays into a lattice and then printed directly upon previous layers. Printed parts were cured in sealed glass vials at $85^{\circ} \mathrm{C}$. After gelation, the wet $\mathrm{GO}$ gels were removed from the glass vials and washed in acetone to remove water from the pores. Supercritical $\mathrm{CO}_{2}$ was used to dry the $\mathrm{GO}$ gels, and they were thermally reduced at $1,050{ }^{\circ} \mathrm{C}$ under nitrogen. Finally, etching in hydrofluoric acid solution was used to remove the silica nanoparticle filler.

Characterization. The dimension and weight of the samples were determined with a caliper with an accuracy of $0.01 \mathrm{~mm}$ and an ultra-micro balance (XP24, Mettler Toledo) with an accuracy of $0.001 \mathrm{mg}$. The relative density was calculated from the measured mass and volume of each specimen. The compressive characteristics of printed specimens were measured using a universal testing machine (Instron 5943) equipped with a $1,000 \mathrm{~N}$ load cell at a nominal rate of $5 \mu \mathrm{m} \mathrm{s}^{-1}$. The Young's modulus was calculated from the initial slope of the unloading stress-strain curves between 0 and $10 \%$ strain ranges ${ }^{56-58}$. Simultaneously, the electrical conductivity was measured by a two-electrode method and two metal wires were used as the current collectors. To optimize the electrical contact between conductive copper face sheets and aerogel, each end of the aerogel was carefully affixed to copper sheet with a thin layer of silver paste. The morphology of the printed samples was observed by optical camera and field-emission SEM. SEM and EDS was performed on a JEOL $7401-\mathrm{F}$ at $10 \mathrm{keV}(20 \mathrm{~mA})$ in secondary electron imaging mode with a working distance of $2-8 \mathrm{~mm}$. Electrical conductivity was measured using the fourprobe method. Textural properties were determined by Brunauer-Emmett-Teller and Barrett-Joyner-Halenda methods using an ASAP 2000 Surface Area Analyzer (Micromeritics Instrument Corporation) via nitrogen porosimetry ${ }^{1}$. Samples of $\sim 0.1 \mathrm{~g}$ were heated to $150^{\circ} \mathrm{C}$ under vacuum $\left(10^{-5}\right.$ Torr $)$ for at least $24 \mathrm{~h}$ to remove all adsorbed species. XRD measurements were performed on a Bruker AXS D8 ADVANCE X-ray diffractometer equipped with a LynxEye 1-dimensional linear Si strip detector. The samples were scanned from 5 to $75^{\circ} 2 \theta$. The step scan parameters were $0.02^{\circ}$ steps and $2 \mathrm{~s}$ counting time per step with a $0.499^{\circ}$ divergence slit and a $0.499^{\circ}$ antiscatter slit. The X-ray source was Ni-filtered Cu radiation from a sealed tube operated at $40 \mathrm{kV}$ and $40 \mathrm{~mA}$. Phases in the samples were identified by comparison of observed peaks to those in the International Centre for Diffraction Data (ICDD PDF2009) powder diffraction database, and also peaks listed in reference articles. Goniometer alignment was ensured using a Brukersupplied $\mathrm{Al}_{2} \mathrm{O}_{3}$ standard.

\section{References}

1. Geim, A. K. \& Novoselov, K. S. The rise of graphene. Nature Mater. 6, 183-191 (2007).

2. Li, D. \& Kaner, R. B. Graphene-based materials. Science 320, 1170-1171 (2008)

3. Geim, A. K. Graphene: status and prospects. Science 324, 1530-1534 (2009).

4. Peigney, A., Laurent, C., Flahaut, E., Bacsa, R. R. \& Rousset, A. Specific surface area of carbon nanotubes and bundles of carbon nanotubes. Carbon 39, 507-514 (2001)

5. Lee, C., Wei, X., Kysar, J. W. \& Hone, J. Measurement of the elastic properties and intrinsic strength of monolayer graphene. Science 321, 385-388 (2008).

6. Balandin, A. A. et al. Superior thermal conductivity of single-layer graphene. Nano Lett. 8, 902-907 (2008).

7. Li, X. et al. Highly conducting graphene sheets and Langmuir-Blodgett films. Nature Nanotech. 3, 538-542 (2008).

8. Eda, G. \& Chhowalla, M. Graphene-based composite thin films for electronics Nano Lett. 9, 814-818 (2009).

9. Eda, G., Fanchini, G. \& Chhowalla, M. Large-area ultrathin films of reduced graphene oxide as a transparent and flexible electronic material. Nature Nanotech. 3, 270-274 (2008).

10. Schedin, F. et al. Detection of individual gas molecules adsorbed on graphene. Nature Mater. 6, 652-655 (2007).

11. Shao, Y. et al. Graphene based electrochemical sensors and biosensors: a review. Electroanalysis 22, 1027-1036 (2010).

12. Machado, B. F. \& Serp, P. Graphene-based materials for catalysis. Catal. Sci. Technol. 2, 54-75 (2012).

13. Sutter, P. W., Flege, J.-I. \& Sutter, E. A. Epitaxial graphene on ruthenium. Nature Mater. 7, 406-411 (2008)

14. Ramanathan, T. et al. Functionalized graphene sheets for polymer nanocomposites. Nature Nanotech. 3, 327-331 (2008).

15. Vickery, J. L., Patil, A. J. \& Mann, S. Fabrication of graphene-polymer nanocomposites with higher-order three-dimensional architectures. Adv. Mater. 21, 2180-2184 (2009).

16. Wang, X., Zhi, L. \& Müllen, K. Transparent, conductive graphene electrodes for dye-sensitized solar cells. Nano Lett. 8, 323-327 (2008).

17. Yoo, E. et al. Large reversible Li storage of graphene nanosheet families for use in rechargeable lithium ion batteries. Nano Lett. 8, 2277-2282 (2008).

18. Menaa, F., Abdelghani, A. \& Menaa, B. Graphene nanomaterials as biocompatible and conductive scaffolds for stem cells: impact for tissue engineering and regenerative medicine. J. Tissue Eng. Regen. Med. doi: 10.1002/term.1910 (2014).

19. Chen, Z. et al. Three-dimensional flexible and conductive interconnected graphene networks grown by chemical vapor deposition. Nature Mater. 10, 424-428 (2011).

20. Xu, Y., Sheng, K., Li, C. \& Shi, G. Self-assembled graphene hydrogel via a onestep hydrothermal process. ACS Nano 4, 4324 (2010).

21 . Bi, H. et al. Low temperature casting of graphene with high compressive strength. Adv. Mater. 24, 5124-5129 (2012).

22. Zhao, J., Ren, W. \& Cheng, H.-M. Graphene sponge for efficient and repeatable adsorption and desorption of water contaminations. J. Mater. Chem. 22, 20197-20202 (2012).

23. Bai, H., Li, C., Wang, X. \& Shi, G. A pH-sensitive graphene oxide composite hydrogel. Chem. Commun. 46, 2376-2378 (2010).

24. Lin, Y., Ehlert, G. J., Bukowsky, C. \& Sodano, H. A. Superhydrophobic functionalized graphene aerogels. ACS Appl. Mater. Interfaces 3, 2200-2203 (2011).

25. Han, Z. et al. Ammonia solution strengthened three-dimensional macro-porous graphene aerogel. Nanoscale 5, 5462-5467 (2013).

26. Cong, H. P., Ren, X. C., Wang, P. \& Yu, S. H. Macroscopic multifunctional graphene-based hydrogels and aerogels by a metal ion induced self-assembly process. ACS Nano 6, 2693-2703 (2012).

27. Chen, W. \& Yan, L. In situ self-assembly of mild chemical reduction graphene for three-dimensional architectures. Nanoscale 3, 3132-3137 (2011).

28. Sun, H. Y., Xu, Z. \& Gao, C. Multifunctional, ultra-flyweight, synergistically assembled carbon aerogels. Adv. Mater. 25, 2554-2560 (2013).

29. Worsley, M. A. et al. Toward macroscale, isotropic carbons with graphenesheet-like electrical and mechanical properties. Adv. Funct. Mater. 24, 4259-4264 (2014).

30. Worsley, M. A. et al. Mechanically robust 3D graphene macroassembly with high surface area. Chem. Commun. 48, 8428-8430 (2012). 
31. Zhang, X. et al. Mechanically strong and highly conductive graphene aerogel and its use as electrodes for electrochemical power sources. J. Mater. Chem. 21, 6494-6497 (2011).

32. Bai, H., Li, C., Wang, X. \& Shi, G. On the gelation of graphene oxide. J. Phys. Chem. C. 115, 5545-5551 (2011).

33. Worsley, M. A. et al. Synthesis of graphene aerogel with high electrical conductivity. J. Am. Chem. Soc. 132, 14067-14069 (2010).

34. Worsley, M. A. et al. High surface area, sp2-cross-linked three-dimensional graphene monoliths. J. Phys. Chem. Lett. 2, 921-925 (2011).

35. Barg, S. et al. Mesoscale assembly of chemically modified graphene into complex cellular networks. Nat. Commun. 5, 4328 (2014).

36. Qiu, L., Liu, J. Z., Chang, S. L., Wu, Y. \& Li, D. Biomimetic superelastic graphene-based cellular monoliths. Nat. Commun. 3, 1241 (2012).

37. Gibson, L. J. \& Ashby, M. F. Cellular Solids: Structure and Properties (Cambridge Univ. Press, 1999).

38. Colombo, P. \& Degischer, H. Highly porous metals and ceramics. Mater. Sci. Technol. 26, 1145-1158 (2010).

39. Schaedler, T. et al. Ultralight metallic microlattices. Science 334, 962-965 (2011).

40. Zheng, X. et al. Ultralight, ultrastiff mechanical metamaterials. Science 344, 1373-1377 (2014)

41. Duoss, E. B. et al. Three-dimensional printing of elastomeric, cellular architectures with negative stiffness. Adv. Funct. Mater. 24, 4905-4913 (2014).

42. Compton, B. G. \& Lewis, J. A. 3D-printing of lightweight cellular composites. Adv. Mater. 26, 5930-5935 (2014).

43. Lewis, J. A. Direct ink writing of 3D functional materials. Adv. Funct. Mater 16, 2193-2204 (2006).

44. Smay, J. E., Gratson, G. M., Shepherd, R. F., Cesarano, J. I. I. I. \& Lewis, J. A. Directed colloidal assembly of 3D periodic structures. Adv. Mater. 14, 1279-1283 (2002)

45. Zhu, C. \& Smay, J. E. Thixotropic rheology of concentrated alumina colloidal gels for solid freeform fabrication. J. Rheol. 55, 655-672 (2011).

46. Lewis, J. A. \& Smay, J. E. in Cellular Ceramics: Structure, Manufacturing, Properties and Applications. (eds Scheffler, M. \& Colombo, P.) 87-100 (Wiley$\mathrm{VCH}, 2006$ ).

47. García-Tuñon, E. et al. Printing in three dimensions with graphene. Adv. Mater. 27, 1688-1693 (2015).

48. Le, L. T., Ervin, M. H., Qiu, H., Fuchs, B. E. \& Lee, W. Y. Graphene supercapacitor electrodes fabricated by inkjet printing and thermal reduction of graphene oxide. Electrochem. Commun. 13, 355-358 (2011).

49. Naficy, S. et al. Graphene oxide dispersions: tuning rheology to enable fabrication. Mater. Horiz. 1, 326-331 (2014).

50. Pekala, R., Alviso, C. \& LeMay, J. Organic aerogels: microstructural dependence of mechanical properties in compression. J. Non-Cryst. Solids 125, 67-75 (1990).

51. Tang, Z. H., Shen, S. L., Zhuang, J. \& Wang, X. Noble-metal-promoted threedimensional macroassembly of single-layered graphene oxide. Angew. Chem. Int. Ed. 49, 4603-4607 (2010).

52. Worsley, M. A., Kucheyev, S. O., Satcher, Jr. J. H., Hamza, A. V. \& Baumann, T. F. Mechanically robust and electrically conductive carbon nanotube foams. Appl. Phys. Lett. 94, 073115 (2009).
53. Kim, K. H., Oh, Y. \& Islam, M. Graphene coating makes carbon nanotube aerogels superelastic and resistant to fatigue. Nature Nanotech. 7, 562-566 (2012).

54. Si, Y., Yu, J., Tang, X., Ge, J. \& Ding, B. Ultralight nanofibre-assembled cellular aerogels with superelasticity and multifunctionality. Nat. Commun. 5, 5802 (2014).

55. Hummers, Jr. W. S. \& Offeman, R. E. Preparation of graphitic oxide. J. Am. Chem. Soc. 80, 1339-1339 (1958).

56. Kan, Q., Yan, W., Kang, G. \& Sun, Q. Oliver-Pharr indentation method in determining elastic moduli of shape memory alloys-a phase transformable material. J. Mech. Phys. Solids 61, 2015-2033 (2013).

57. Oliver, W. C. \& Pharr, G. M. An improved technique for determining hardness and elastic modulus using load and displacement sensing indentation experiments. J. Mater. Res. 7, 1564-1583 (1992).

58. Oliver, W. C. \& Pharr, G. M. Measurement of hardness and elastic modulus by instrumented indentation: Advances in understanding and refinements to methodology. J. Mater. Res. 19, 3-20 (2004).

\section{Acknowledgements}

This work was supported by Lawrence Livermore National Laboratory under the auspices of the US Department of Energy under Contract DE-AC52-07NA27344, through LDRD award 14-SI-004 and 13-LW-099. We thank Tim Ford for optical image acquisition.

\section{Author contributions}

C.Z., E.B.D., J.D.K., C.M.S. and M.A.W. conceived and designed experiments. C.Z., M.A.W., T.Y.-J.H. and A.M.G. prepared samples. M.A.W. and C.Z. were involved in electrical analysis. M.A.W. performed SEM, Raman, XRD, EDS and surface area analysis. C.Z. performed rheological and mechanical experiments. M.A.W., C.Z., E.B.D. and C.M.S. were mainly responsible for preparing the manuscript with further inputs from other authors. All authors discussed the results and commented on the manuscript.

\section{Additional information}

Supplementary Information accompanies this paper at http://www.nature.com/ naturecommunications

Competing financial interests: The authors declare no competing financial interests.

Reprints and permission information is available online at http://npg.nature.com/ reprintsandpermissions/

How to cite this article: Zhu, C. et al. Highly compressible 3D periodic graphene aerogel microlattices. Nat. Commun. 6:6962 doi: 10.1038/ncomms7962 (2015).

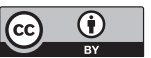

This work is licensed under a Creative Commons Attribution 4.0 International License. The images or other third party material in this article are included in the article's Creative Commons license, unless indicated otherwise in the credit line; if the material is not included under the Creative Commons license, users will need to obtain permission from the license holder to reproduce the material. To view a copy of this license, visit http://creativecommons.org/licenses/by/4.0/ 